\title{
Short communication: Lysine retained among 2 lipid-coated lysine products after exposure to alfalfa or corn silage with different amounts of acidity
}

\author{
J. N. Reiners, J. E. Held, C. L. Wright, and D. W. Brake ${ }^{1}$ \\ Department of Animal Science, South Dakota State University, Brookings 57007
}

\section{ABSTRACT}

We conducted 2 experiments to determine lysine loss from 2 lipid-coated lysine products after mixing with silage. In our first experiment, we mixed 2 lipid-coated lysine products, crystalline lysine or crystalline lysine and amounts of lipid identical to amounts included in lipid-coated lysine products, with alfalfa or corn silage that had 2 different amounts of acidity. Lysine appeared to disassociate from lipid-coated lysine products in a nonlinear manner after mixing with either alfalfa or corn silage at different amounts of acidity. Additionally, silage source and acidity affected amounts of lysine released from lipid-coated lysine products after mixing. In a corresponding experiment, in vitro estimates of lysine available to ruminal microbiota after mixing with alfalfa or corn silage at different amounts of acidity were measured by ammonia release. In vitro measures were conducted with or without monensin to allow estimates of effects of monensin on amounts of lysine released from the 2 lipid-coated lysine products. It is unclear whether in vitro estimates of lysine fermentation from lipid-coated lysine are truly reflective of ruminal degradation of lysine from lipid-coated lysine because amounts of time needed to measure differences between different lysine sources were greater than typical estimates of mean ruminal particulate retention time. Nonetheless, monensin apparently reduced ammonia release from lysine, but ammonia release from lipid-coated lysine did not differ from crystalline lysine. Clearly, methods of manufacture together with physical and chemical characteristics of diet can affect amounts of lysine provided from lipid-coated lysine products to ruminants.

Key words: acidity, cattle, ruminally protected lysine, silage

Received August 18, 2017.

Accepted January 21, 2018

${ }^{1}$ Corresponding author: derek.brake@sdstate.edu

\section{Short Communication}

To be an effective source of metabolizable AA, ruminally protected AA products must withstand mixing, innate chemical characteristics of the diet, mastication, and ruminal fermentation (Papas and Wu, 1997). Ji et al. (2016) reported that mechanical mixing, diet moisture content, and increased amounts of time exposed to feed decreased lysine retained in several lipid-coated lysine products (LCLys). Hydrolysis of triglycerides to fatty acids and glycerol can be catalyzed by acid (Bender et al., 1961; Carey, 2003), and acid-catalyzed hydrolysis is greater in environments with large water concentrations (Le Chatelier, 1884; Bender et al., 1961; Carey, 2003). Thus, it is possible that diets containing large amounts of silage and, concomitantly, large amounts of water and organic acids may affect amounts of AA retained by LCLys. Our objective in this work was to determine effects of acidity and silage source on lysine retained by 2 LCLys products and to estimate ruminal degradation of lysine retained by LCLys after mixing with alfalfa or corn silage with different amounts of acidity. All procedures involving the use of animals were approved by the South Dakota State University Institutional Animal Care and Use Committee.

In an initial experiment we evaluated amounts of lysine retained by 2 LCLys products after exposure to alfalfa or corn silage with different $\mathrm{pH}$ levels. Prior to in situ incubation of LCLys in silage, alfalfa (50\% DM; $741 \mathrm{~kg}$ ) was collected, wilted, and chopped (average particle size $=0.81 \mathrm{~cm})$ with a hammer mill (Patriot Pro-Series model CSV-3090H; Patriot Products Inc., Pewaukee, WI $)$ before it was packed $(0.7 \pm 0.05 \mathrm{~kg} / \mathrm{L})$ into 2 miniature silos $(121 \mathrm{~L})$ and ensiled for $169 \mathrm{~d}$. Similarly, freshly chopped (average particle size $=0.79$ $\mathrm{cm})$ corn plants $(46 \% \mathrm{DM} ; 334 \mathrm{~kg})$ were collected and packed $(0.6 \pm 0.05 \mathrm{~kg} / \mathrm{L})$ into 2 miniature silos $(121 \mathrm{~L})$ and ensiled for $154 \mathrm{~d}$.

After ensiling, corn silage $(\mathrm{pH}=3.7 \pm 0.1)$ and alfalfa silage $(\mathrm{pH}=4.4 \pm 0.1)$ from each of the 2 silos were composited by silage type, and $\mathrm{pH}$ was measured immediately before in situ incubation of LCLys in silage. The $\mathrm{pH}$ of each composited silage was measured 
as described by Buchanan-Smith and Yao (1981). After initial $\mathrm{pH}$ measurement, silage $\mathrm{pH}$ was adjusted by mixing (model 2030, Marion Mixer, Marion, IA) 10\% (wt/wt) $\mathrm{NaOH}$ for 5 min to achieve the appropriate acidity. Acidity in an aliquot of corn silage $(11 \mathrm{~kg}$ of $\mathrm{DM}$ ) was modified to be similar to the initial $\mathrm{pH}$ of alfalfa silage $(\mathrm{pH}=4.6)$ by addition of $1.55 \mathrm{~kg}$ of $\mathrm{NaOH}$. Subsequently, an aliquot of alfalfa silage $(9.5 \mathrm{~kg}$ of DM) was mixed with $2.80 \mathrm{~kg}$ of $\mathrm{NaOH}$ to increase $\mathrm{pH}$ to 6.8. Additionally, another aliquot of corn silage $(11 \mathrm{~kg}$ of DM) was mixed with $3.15 \mathrm{~kg}$ of $\mathrm{NaOH}$ to achieve a similar $\mathrm{pH}(\mathrm{pH}=6.9)$ to alfalfa silage with added $\mathrm{NaOH}$. Modification of silage $\mathrm{pH}$ allowed for evaluation of effects of acidity on lysine loss from LCLys by creating acidic corn silage $(\mathrm{pH}=4.6)$ and alfalfa silage $(\mathrm{pH}$ $=4.4)$ and more neutral corn silage $(\mathrm{pH}=6.9)$ and alfalfa silage $(\mathrm{pH}=6.8)$.

Samples $(4 \mathrm{~g})$ of 2 LCLys products were placed in duplicate polyester bags $(10 \times 20 \mathrm{~cm}$, pore size $=50$ $\mu \mathrm{m}$; Dacron, Ankom Technology, Fairport, NY) and heat sealed before placement in silage. One LCLys product (LP; LysiPearl, Kemin Industries, Des Moines, IA) consisted of $47.5 \%$ lysine- $\mathrm{HCl}$ and $52.5 \%$ lipid and was manufactured by embedding lysine- $\mathrm{HCl}$ into a lipid matrix. The second LCLys (USA; USA Lysine, Kemin Industries) consisted of $65 \%$ lysine- $\mathrm{HCl}$ and was manufactured by extrusion of lysine- $\mathrm{HCl}$ and lipid into small particles and subsequently coating lysine lipid particles with multiple layers of a lipid matrix. Lysine- $\mathrm{HCl}(4 \mathrm{~g})$ alone served as a negative control. Additionally, lysine$\mathrm{HCl}$ and the same lipid used to manufacture LP and USA (58.1\% palmitic acid, $39.2 \%$ stearic acid, $1.3 \%$ myristic acid, $0.5 \%$ arachidic acid, $0.3 \%$ lauric acid, $0.2 \%$ heptadecanoic acid, $0.1 \%$ lignoceric acid, $0.1 \%$ linoleic acid, $0.1 \%$ pentadecylic acid) were added separately to the same polyester bags in amounts identical to either LP or USA and served as controls to evaluate manufacturing method. After silage acidity was adjusted, replicate polyester bags were hand mixed with corn silage $(22.7 \mathrm{~kg})$ or alfalfa silage $(11.4 \mathrm{~kg})$ in a plastic container $(191 \mathrm{~L}$; length $=108.6 \mathrm{~cm}$; width $=55.9 \mathrm{~cm}$; height $=45.7 \mathrm{~cm}$ ) and immediately removed (i.e., 0 h) or incubated for 6,12 , or $24 \mathrm{~h}$ in a temperaturecontrolled room $\left(20^{\circ} \mathrm{C}\right)$. At the appropriate time, bags were removed and rinsed with $5 \mathrm{~L}$ of cold tap water per side over a $40-\mu \mathrm{m}$ screen. After rinsing, samples were frozen $\left(-20^{\circ} \mathrm{C}\right)$ and lyophilized before removal from polyester bags.

Lipid-associated lysine content was analyzed from lyophilized samples as described by Brake et al. (2013). Linear and nonlinear models were used to predict rates of lysine disassociation from LCLys after mixing with silage. Linear estimates of lysine disassociation were calculated as the slope of the regression line of the natural logarithm of lipid-associated lysine in LCLys versus incubation time (Mathers and Miller, 1981; Bach et al., 1998). Additionally, we evaluated a first-order kinetic model to predict rates of lysine disassociation (Ørskov and McDonald, 1979). The first-order kinetic model calculated lysine disassociation as the sum of the proportion of lysine that immediately disassociates from LCLys (ID) and the product of the proportion of lysine that is potentially dissociable (slowly disassociates; SD). The amount of lysine disassociation from SD was calculated as 1 minus the logarithm of the product of the hourly rate of disassociation $\left(\mathbf{K}_{\mathbf{d}}\right)$ and time. The equation was fitted using the Marquardt method for iterative, nonlinear, least squares estimation in SAS (SAS Institute Inc., Cary, NC). Incorporating lag time into the model did not seem appropriate from the shape of the degradation curves (Figure 1). Because a coefficient of determination is not readily defined in nonlinear regression, we calculated pseudocoefficients of determination as 1 - (sum of squared residuals/corrected total sum of squares). Extent of lysine disassociation was calculated as the sum of ID and the product of SD and the quotient of $K_{d}$ and $K_{d}$ plus the rate of diet intake (Ørskov and McDonald, 1979). Because rate of diet intake was assumed to be constant across time (Hart et al., 2014; Yuan et al., 2015), rate of diet intake was calculated as the reciprocal of $8,12,16$, and 24 $\mathrm{h}$, respectively. Subsequently, amounts of lysine that remained associated with each LCLys product were calculated as 1 - extent of lysine disassociation.

Both the linear and nonlinear models were tested for goodness of fit by the lack-of-fit test described by Sokal and Rohlf (1969). Appropriateness of the linear model was evaluated by plotting residuals versus fitted values, and coefficients of determination were calculated with the REG procedure of SAS. Pool sizes (ID, SD, and lysine not disassociated) and $\mathrm{K}_{\mathrm{d}}$ were analyzed as a completely randomized design using the MIXED procedure of SAS. The model contained effects of LCLys, silage type, $\mathrm{pH}$, and all interactions.

We conducted an additional experiment to estimate ruminal degradation of lipid-associated lysine in each LCLys product with or without monensin. Samples (4 g) of LP and USA were placed in polyester bags (10 $\times 20 \mathrm{~cm}$; pore size $=50 \mu \mathrm{m})$ and hand mixed with an aliquot of the same alfalfa silage $(\mathrm{pH}=4.4 ; 11.4 \mathrm{~kg})$ and corn silage $(\mathrm{pH}=3.7 ; 22.7 \mathrm{~kg})$ used in Experiment 1 before addition of $\mathrm{NaOH}$. Polyester bags containing LP and USA were removed after $30 \mathrm{~min}$, rinsed with 5 $\mathrm{L}$ of cold tap water per side over a $40-\mu \mathrm{m}$ screen, frozen $\left(-20^{\circ} \mathrm{C}\right)$, and subsequently lyophilized inside polyester bags. 


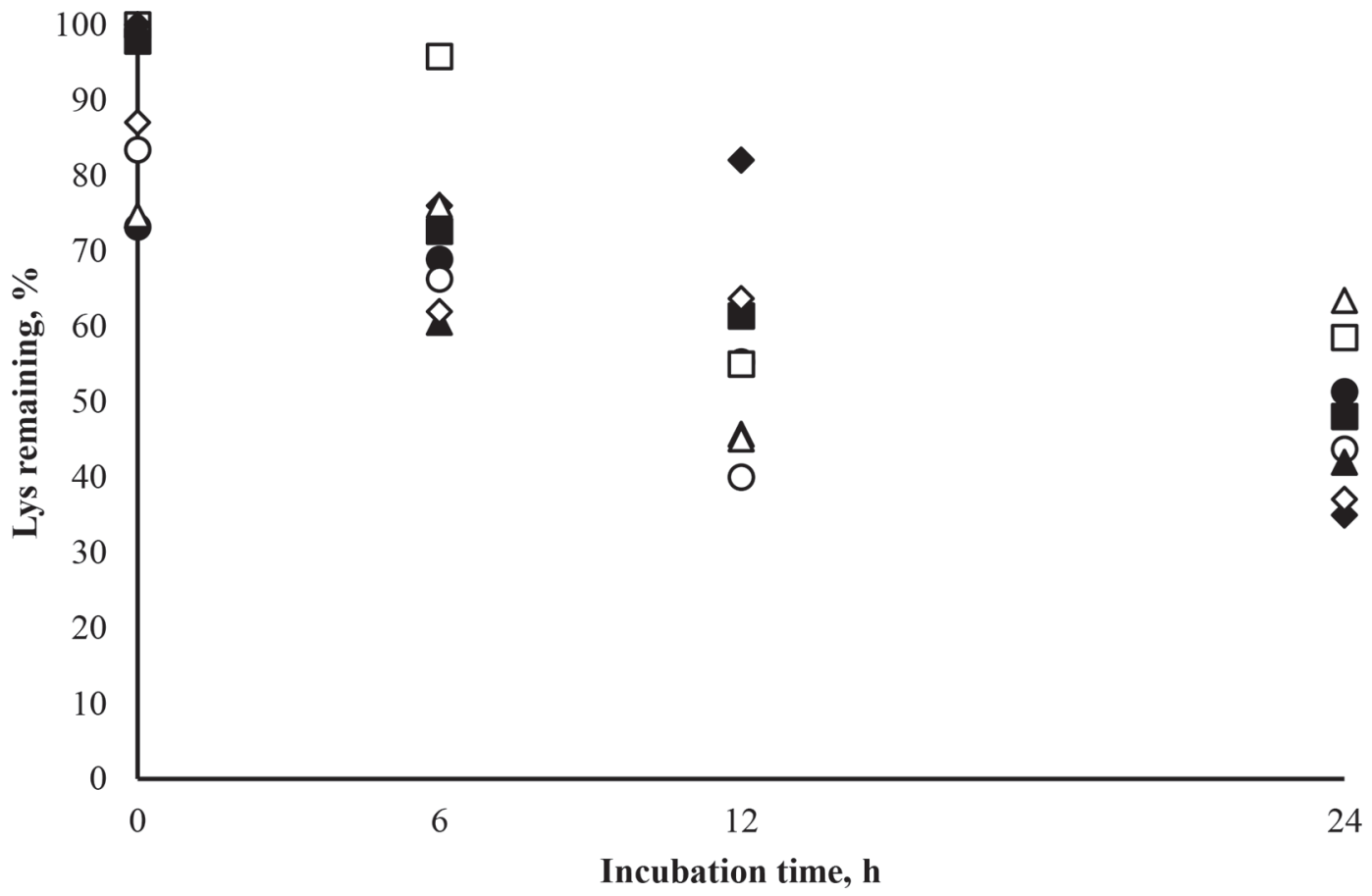

Figure 1. Lysine loss from lipid-coated lysine products after exposure to acidic $(\mathrm{pH}=4.6)$ or neutral $(\mathrm{pH}=6.8)$ alfalfa or corn silage. LP in acidic alfalfa silage, $\mathbf{\square}$; USA in acidic alfalfa silage, $\mathbf{0}$; LP in neutral alfalfa silage, $\square$; USA in neutral alfalfa silage, $\bigcirc$; LP in acidic corn silage, $\checkmark$; USA in acidic corn silage, $\mathbf{\Delta}$; LP in neutral corn silage, $\diamond$; USA in neutral corn silage, $\Delta$. LP $=$ LysiPearl (Kemin Industries, Des Moines, IA); USA = USA Lysine (Kemin Industries).

Fresh ruminal contents from 2 ruminally cannulated cows fed a corn-based diet were collected $4 \mathrm{~h}$ after feeding. The ingredient composition $(\% \mathrm{DM})$ of the diet was $35.3 \%$ dry rolled corn, $25.2 \%$ corn silage, $15.2 \%$ alfalfa hay, $10.0 \%$ soybean hulls, $9.0 \%$ soybean meal, $1.0 \%$ calcium soaps of long-chain fatty acids (MegaLacR; Church and Dwight Co., Princeton, NJ), $0.4 \%$ monocalcium phosphate, $0.3 \%$ LP, $0.3 \%$ trace mineralized salt (contained $98.9 \% \mathrm{NaCl}, 0.4 \% \mathrm{Zn}, 0.16 \% \mathrm{Fe}$, $0.12 \% \mathrm{Mn}, 0.029 \% \mathrm{Cu}, 0.01 \% \mathrm{I}$, and $0.004 \% \mathrm{Co}), 0.2 \%$ magnesium oxide, $0.05 \%$ of a trace mineral (contained $5.15 \% \mathrm{Zn}, 2.86 \% \mathrm{Mn}, 1.80 \% \mathrm{Cu}, 0.18 \%$ Co; Availa 4; Zinpro Corp., Eden Prairie, MN), and $0.05 \%$ of a vitamin and Se premix (provided 3,300 IU of vitamin $\mathrm{A} / \mathrm{kg}, 2,250 \mathrm{IU}$ of vitamin $\mathrm{D} / \mathrm{kg}, 35 \mathrm{IU}$ of vitamin $\mathrm{E} /$ $\mathrm{kg}$, and $0.06 \mathrm{mg}$ of $\mathrm{Se} / \mathrm{kg}$ ). Ruminal contents were strained through 4 layers of cheesecloth into thermally insulated containers and immediately transported to the laboratory. Ruminal fluid was centrifuged $(150 \times g$ at $4^{\circ} \mathrm{C}$ ) for $5 \mathrm{~min}$, and the supernatant layer was used as ruminal bacterial inocula. Ruminal bacterial inocula $(10 \mathrm{~mL})$ and $40 \mathrm{~mL}$ of McDougall's buffer (McDougall, 1948) with urea (13.3 $\mathrm{m} M$ urea) was dispensed into duplicate plastic centrifuge tubes $(3.4 \mathrm{~mm} \times$ $16.2 \mathrm{~mm}$; Nalge Nunc International Corp., Rochester,
$\mathrm{NY}$ ) and flushed with $\mathrm{CO}_{2}$. Treatments consisted of additions of monensin ( 0 or $5 \mathrm{mg} / \mathrm{L}$ of monensin; lot no. M5273; Sigma-Aldrich, St. Louis, MO) and lysine $(30 \mathrm{mM})$ from LP or USA exposed to silage for 30 min, lysine- $\mathrm{HCl}$, or lysine- $\mathrm{HCl}$ and lipid in amounts identical to LP or USA. Monensin was added as 25 $\mu \mathrm{L}$ of a monensin and ethanol solution prepared by dissolving $100 \mathrm{mg}$ of monensin in $10 \mathrm{~mL}$ of anhydrous ethanol. Ruminal inocula and McDougall's buffer alone served as the negative control. Fermentation tubes were capped with butyl rubber stoppers fitted with Bunsen valves, vortexed, and incubated at $39^{\circ} \mathrm{C}$ for $48 \mathrm{~h}$. At 0 , $6,12,24,30,36,42$, and $48 \mathrm{~h}$, fermentation tubes were vortexed and an aliquot of fluid $(1 \mathrm{~mL})$ was removed to a microcentrifuge tube and acidified with $0.2 \mathrm{~mL}$ of $1 \mathrm{M} \mathrm{HCl}$ before freezing $\left(-20^{\circ} \mathrm{C}\right)$. Subsequently, each aliquot of fermentation fluid was analyzed for ammonia concentration (Broderick and Kang, 1980).

Ammonia concentration in fermentation fluid was analyzed as a randomized complete block design with the MIXED procedure of SAS. The model included effects of time, treatment, monensin, and all interactions, and effect of cow was random. Denominator degrees of freedom were calculated by the Kenward-Roger adjustment (Kenward and Roger, 1997). Linear and quadratic 
effects of time were determined by orthogonal contrasts. When the $F$-statistic was significant $(P \leq 0.05)$, means were separated using a Student's t-test.

It is important that mathematical models be tested for goodness of fit before use in predicting nutrient supplies to ruminants (Bach et al., 1998). As expected, the proportion of lysine that instantly disassociated from the negative control (lysine- $\mathrm{HCl}$ alone) and each manufacturing control (i.e., lipid and lysine- $\mathrm{HCl}$ in amounts identical to LP or USA) were complete $(100 \pm 2.5 \%)$; therefore, these data were excluded from estimations of lysine disassociation from LCLys. We calculated a moderate coefficient of determination $\left(\mathrm{R}^{2}=0.56\right)$ when we linearly regressed the natural logarithm of residual lysine in the LCLys products and incubation time in silage. Residuals should be equally distributed about a fitted line without any pattern when linear estimations are appropriate; however, linear estimation of lysine disappearance from LCLys incubated in silage resulted in a U-shaped distribution of residuals (data not shown). Additionally, the linear model was rejected by the lack-of-fit test (Sokal and Rohlf, 1969). Alternatively, estimates of lysine disappearance from LCLys incubated in silage by a first-order kinetics model appeared to have a better fit (average pseudo $R^{2}=0.87$ ) than linear estimates and did not fail the lack-of-fit test.

Ji et al. (2016) reported that mixing decreased lysine retained by LCLys and that large amounts of lysine were lost from LP and USA. Generally, the manufacturing method of LCLys contributes to a distribution of lysine and lipid throughout the lysine-lipid particle. Differences in ruminal degradation of protein are related to different physical characteristics of proteins (e.g., hydropathy, solubility, molecular charge). Similar to proteins, differences in physical characteristics among lysine molecules in lysine-lipid particles may allow for different rates of lysine disassociation from LCLys. It is reasonable that lysine located at the peripheral surface of the lysine-lipid particle more rapidly disassociates compared with lysine embedded nearer to the core. Lipids can be hydrolyzed when exposed to environments with acids and water (Le Chatelier, 1884). Hydrolysis of the outer layer of lipid in the lysine-lipid particle could allow for subsequent solubilization of lysine nearer to the core of the lysine-lipid particle. However, complete loss of lysine from manufacturing controls indicates that addition of lysine to silage without some form of lipid encapsulation would likely not provide metabolizable lysine to ruminants.

We did not observe an interaction among product, silage, and $\mathrm{pH}$ on lysine disassociation from LCLys, and lysine disassociation kinetics are reported in Table 1. Amounts of lysine that immediately disassociated from each LCLys product were different between alfalfa silage and corn silage at different amounts of acidity (silage $\times \mathrm{pH} ; P=0.02$ ). Specifically, lysine that immediately disassociated from each LCLys product increased in corn silage as $\mathrm{pH}$ increased, but amounts of lysine that immediately disassociated from each LCLys product in alfalfa silage did not differ and were numerically less when alfalfa silage was nearer a neutral pH. Ji et al. (2016) reported that diet moisture content had no effect on amounts of lysine that disassociated from the same LCLys products evaluated in this study. Overall, lysine that immediately disassociated from USA $(15.7 \%)$ was greater $(P=0.04)$ than that from LP (4.9\%). Differences in the manufacture of LCLys may affect initial disassociation of lysine from LCLys depending on silage type and $\mathrm{pH}$.

Amounts of lysine that slowly disassociated from each LCLys product were less in corn silage near a neutral $\mathrm{pH}$ compared with acidic corn silage, and amounts of lysine that slowly disassociated from alfalfa silage were not affected by acidity (silage $\times \mathrm{pH} ; P=0.02$ ). More than twice as much lysine slowly disassociated from LCLys exposed to acidic corn silage than from LCLys exposed to neutral corn silage $(P=0.01)$. A greater extent of acid-catalyzed hydrolysis of triglyceride in LCLys could allow greater amounts of lysine in the lysine-lipid particle to be dissolved by water in silage. However, acidity did not affect $(P=0.40)$ lysine that slowly disassociated from LCLys mixed in alfalfa silage. Perhaps greater abrasion of LCLys by alfalfa silage during mixing (Reiners et al., 2017) reduced the effect of acidity on amount of lysine that slowly disassociated from each LCLys product.

Greater amounts of lysine slowly disassociated from LP $(67.5 \%)$ compared with USA $(46.7 \% ; P=0.01)$. Clearly, differences in manufacture of LCLys products can affect the amount of lysine that disassociates after mixing with silage. It is possible that differences in manufacture contributed to a more uniform dispersion of lysine across the lysine-lipid particle in LP compared with a greater amount of lysine nearer the core in USA. Greater amounts of slowly dissociable lysine in LP compared with USA may be explained if lysine nearer to the surface of each LCLys product disassociated sooner after mixing with silage than lysine nearer the core of LCLys; however, LP had smaller amounts of lysine immediately disassociate.

Lysine that did not disassociate by $24 \mathrm{~h}(32.5 \pm$ $5.4 \%$; Table 1) did not differ between type of LCLys $(P=0.30)$ and was not affected by silage $(P=0.64)$. However, greater acidity reduced amounts of lysine that did not disassociate from each LCLys product by almost 49\%. Ji et al. (2016) reported that less lysine disassociated from LP and USA $24 \mathrm{~h}$ after mixing 


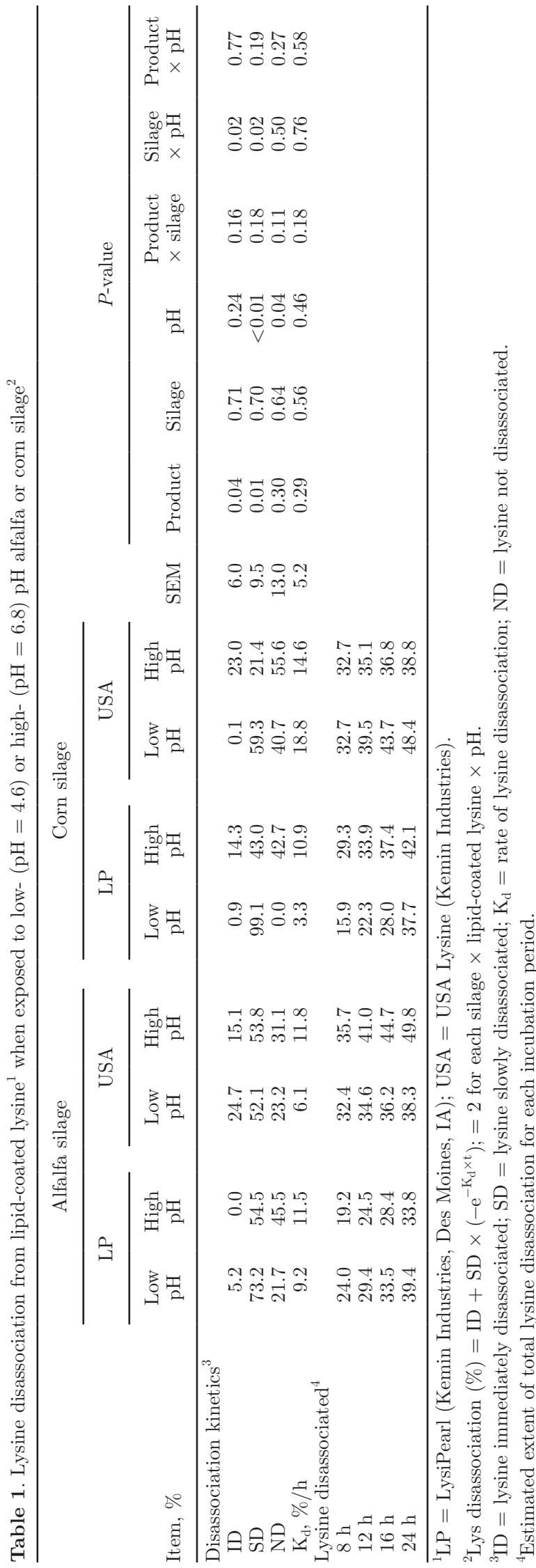

in TMR compared with our estimates. Together, the observations of $\mathrm{Ji}$ et al. (2016) and our data provide evidence that lysine in some LCLys may be affected by a complex interaction of forces that include physical and chemical characteristics of feed.

Rate of lysine disassociation from LCLys was 10.8\%/h, and we observed no differences between each LCLys product or effect of silage source or acidity on rate of lysine disassociation $(P \geq 0.21)$. This seems to suggest that differences in LCLys manufacture and effects of silage and acidity had a greater effect on amounts of lysine able to slowly disassociate from LCLys products than rate of lysine disassociation.

Estimates of extent of lysine disassociation from LP and USA reflected effects of silage source and acidity among the 2 sources of LCLys (Table 1). Estimates of extent of lysine disassociation from each LCLys product were almost 14 and $22 \%$ less for an 8-h feeding interval than for 12- and 16-h feeding intervals, respectively. Further, the average estimate of extent of lysine disassociation from LCLys during the 8-h feeding interval was $31 \%$ less compared with a 24 -h feeding interval.

There was no interaction of lysine source, monensin, and time $(P=1.00)$ on apparent amounts of ammonia released from lysine. Ammonia concentration in incubation fluid (Table 2) increased (quadratic; $P<0.01$ ) with greater amounts of time. However, apparent ammonia release from in vitro cultures containing lysine was not different from cultures containing no lysine until $36 \mathrm{~h}$; at $36 \mathrm{~h}$ after fermentations were initiated, all cultures containing added lysine had greater ammonia content than cultures containing no added lysine. After $42 \mathrm{~h}$ of incubation, apparent ammonia release from lysine was greatest from USA and when lipid and lysine in amounts identical to LP were provided to cultures; LP, lysine- $\mathrm{HCl}$, and lipid and lysine in amounts identical to USA were intermediate. Among cultures with added lysine, values for LP were greatest and lysine$\mathrm{HCl}$ were least after $48 \mathrm{~h}$ of incubation, whereas USA and amounts of lipid and lysine identical to either USA or LP were intermediate. Interestingly, after 48-h incubations, ammonia concentration was less in cultures containing monensin. Further, apparent amounts of ammonia released from LP and USA were not affected by mixing with either alfalfa or corn silage before in vitro incubations.

In this study we were unable to detect differences in ammonia release from added amounts of lysine until 36 h. Even though ammonia content of cultures containing added lysine increased at a quadratic rate after 36 $\mathrm{h}$, amounts of ammonia released after $48 \mathrm{~h}$ reflected only $61 \%$ of $\mathrm{N}$ contained in added amounts of lysine. Similarly, Elwakeel et al. (2012) reported that $55 \%$ of $\mathrm{N}$ from added amounts of lysine was released after $48-\mathrm{h}$ 
Table 2. In vitro ammonia $(\mathrm{m} M)$ release from lipid-coated lysine ${ }^{1}$ products, their analogs, or lysine- $\mathrm{HCl}^{2}$

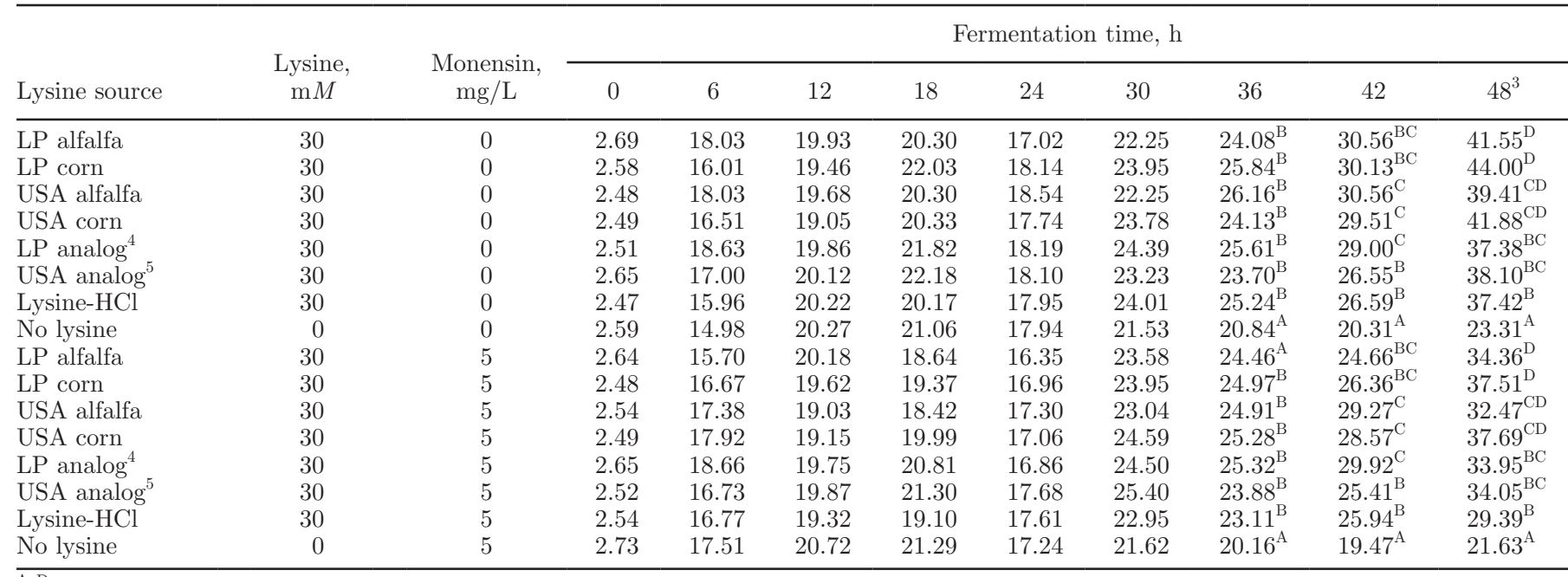

A-D Means within a column with different superscripts differ significantly $(P<0.05)$.

${ }^{1} \mathrm{LP}=$ LysiPearl (Kemin Industries, Des Moines, IA); USA = USA Lysine (Kemin Industries).

${ }^{2}$ Effect of hour was quadratic.

${ }^{3}$ Effect of monensin was significant $(P<0.01)$ and decreased ammonia concentration.

${ }^{4} \mathrm{Lysine}-\mathrm{HCl}$ and lipid added to in vitro cultures in amounts identical to LP.

${ }^{5} \mathrm{Lysine}-\mathrm{HCl}$ and lipid added to in vitro cultures in amounts identical to USA.

incubations with mixed ruminal fluid. Ammonia release after $48 \mathrm{~h}$ of incubation was greatest from cultures that had added lysine and lipid. Additionally, monensin reduced ruminal ammonia release after $48 \mathrm{~h}$ of incubation. Chen and Russell (1989) reported that monensin can inhibit gram-positive bacteria that rapidly ferment lysine. Even though monensin reduced apparent ammonia release from lysine in mixed ruminal cultures by almost $16 \%$ after $48 \mathrm{~h}$ in our study, levels of ammonia release greatly exceeded amounts of ammonia released from lysine in mixed ruminal cultures that were reported by Russell (2005). Extrapolation of in vitro data to the rumen requires caution (Russell, 2006), and it remains equivocal whether differences observed in the model described by Russell (2006) accurately predict relative differences among lysine degradation in the rumen.

These data indicate that both physical and chemical characteristics of diet can affect lysine provided to ruminants from LCLys products with observed low relative bioavailability values (Reiners et al., 2017). It is likely that large amounts of lysine are lost from LP and USA when mixed in silage-based diets before they are consumed by ruminants. Additionally, in vitro estimates seem to suggest that a portion of lipid-associated lysine in LP and USA mixed with silage is available for ruminal fermentation. Further research is needed on bioavailability of lysine from LCLys after exposure of LCLys to various feeding conditions.

\section{ACKNOWLEDGMENTS}

This project was supported by Kemin Industries (Des Moines, IA). This project is a contribution from the South Dakota Agricultural Experiment Station (Brookings).

\section{REFERENCES}

Bach, A., M. D. Stern, N. R. Merchen, and J. K. Drackley. 1998 Evaluation of selected mathematical approaches to the kinetics of protein degradation in situ. J. Anim. Sci. 76:2885-2893.

Bender, M. L., H. Matsui, R. J. Thomas, and S. W. Tobey. 1961. The concurrent alkaline hydrolysis and isotopic oxygen exchange of several alkyl benzoates and lactones. J. Am. Chem. Soc. 83:41934196.

Brake, D. W., E. C. Titgemeyer, M. J. Brouk, C. A. Macgregor, J. F. Smith, and B. J. Bradford. 2013. Availability to lactating dairy cows of methionine added to soy lecithins and mixed with a mechanically extracted soybean meal. J. Dairy Sci. 96:3064-3074.

Broderick, G. A., and J. H. Kang. 1980. Automated simultaneous determination of ammonia and total amino acids in ruminal fluid and in vitro media. J. Dairy Sci. 63:64-75.

Buchanan-Smith, J. G., and Y. T. Yao. 1981. Effect of additives containing lactic acid bacteria and/or hydrolytic enzymes with an antioxidant upon the preservation of corn or alfalfa silage. Can. J. Anim. Sci. 61:669-680.

Carey, F. A. 2003. Carboxylic acid derivatives: Nucleophilic acyl substitution. Pages 830-885 in Organic Chemistry. 5th ed. S. R. Oberbroeckling, ed. McGraw-Hill, New York, NY.

Chen, G., and J. B. Russell. 1989. More monensin-sensitive, ammoniaproducing bacteria from the rumen. Appl. Environ. Microbiol. 55:1052-1057.

Elwakeel, E. A., E. C. Titgemeyer, B. R. Faris, D. W. Brake, A. M. Nour, and M. E. A. Nasser. 2012. Hydroxymethyl lysine is a source of bioavailable lysine for ruminants. J. Anim. Sci. 90:3898-3904. 
Hart, K. D., B. W. McBride, T. F. Duffield, and T. J. DeVries. 2014. Effect of frequency of feed delivery on the behavior and productivity of lactating dairy cows. J. Dairy Sci. 97:1713-1724.

Ji, P., H. A. Tucker, R. E. Clark, M. Miura, and C. S. Ballard. 2016 Short communication: Effect of on-farm feeding practices on rumen protected lysine products. J. Dairy Sci. 99:1242-1246.

Kenward, M. G., and J. H. Roger. 1997. Small sample inference for fixed effects from restricted maximum likelihood. Biometrics 53:983-997.

Le Chatelier, H. L. 1884. On a general statement of the laws of chemical equilibria. Comptes Rendus Acad. Sci. 99:786-789.

Mathers, J. C., and E. L. Miller. 1981. Quantitative studies of food protein degradation and the energetic efficiency of microbial protein synthesis in the rumen of sheep given chopped Lucerne and rolled barley. Br. J. Nutr. 45:587-604.

McDougall, E. I. 1948. Studies on ruminant saliva I. The composition and output of sheep's saliva. Biochem. J. 43:99-109.

Ørskov, E. R., and I. McDonald. 1979. The estimation of protein degradability in the rumen from incubation measurements weighted according to rate of passage. J. Agric. Sci. 92:499-503.
Papas, A., and S. H. Wu. 1997. Rumen-stable delivery systems. Adv. Drug Deliv. Rev. 28:323-334.

Reiners, J. N., J. E. Held, C. L. Wright, Q. Qiao, K. M. Reza, and D. W. Brake. 2017. Lysine bioavailability among 2 lipid coated lysine products after exposure to silage. Transl. Anim. Sci. 1:311-319.

Russell, J. B. 2005. Enrichment of fusobacteria from the rumen that can utilize lysine as an energy source for growth. Anaerobe 11:177184 .

Russell, J. B. 2006. Factors affecting lysine degradation by ruminal fusobacteria. FEMS Microbiol. Ecol. 56:18-24.

Sokal, R. F., and F. J. Rohlf. 1969. Comparisons of Regression Lines. Biometry. Freeman, San Francisco, CA.

Yuan, K., T. Liang, M. B. Muckey, L. G. D. Mendonça, L. E. Hulbert, C. C. Elrod, and B. J. Bradford. 2015. Yeast product supplementation modulated feeding behavior and metabolism in transition dairy cows. J. Dairy Sci. 98:532-540. 